\title{
Vision-based Vehicle Body Slip Angle Estimation with Multi-rate Kalman Filter considering Time Delay
}

\author{
Yafei Wang, Binh Minh Nguyen, \\ Palakon Kotchapansompote, \\ Department of Electrical Engineering, \\ The University of Tokyo, Tokyo, 113-0033, Japan \\ E-mail: \{wang, minh, palakon\}@hori.k.u-tokyo.ac.jp
}

\author{
Hiroshi Fujimoto, and Yoichi Hori \\ Department of Advanced Energy, \\ Graduate School of Frontier Sciences, \\ The University of Tokyo, Chiba, 227-8561, Japan \\ E-mail: \{fujimoto, hori\}@k.u-tokyo.ac.jp
}

\begin{abstract}
Body slip angle is one of the most important information for vehicle motion control; as specific sensors for body slip angle measurement are expensive, it is necessary to investigate estimation methods using existing popular sensors such as gyro sensor, encoder, camera, etc. For EV (electric vehicle), in particular, the motor response is several milliseconds which enables high performance control with short control period; fast signal feedback is consequently desired. Nevertheless, the sampling rate of a normal camera is much slower compared with other kinds of onboard sensors and the time delay caused by image processing cannot be neglected. In this paper, the two problems are solved using a multi-rate Kalman filter with measurement delay included; the estimated body slip angle can be updated every $1 \mathrm{~ms}$. First of all, vehicle model and visual model are explained followed with experimental setup introduction; then, real-time image processing techniques are briefly introduced; and then, singlerate and multi-rate Kalman filters considering time delay are designed to estimate body slip angle; finally, conclusion and further works are presented.
\end{abstract}

\section{INTRODUCTION}

The development of EVs is a promising solution for green transportation, and lots of researches have focused on energy management and power electronics design; on the other hand, motion control for EV is also an important topic [1]. For vehicle motion control system, body slip angle is known to be one of the key enablers; but existing products for direct body slip angle measurement such as optical sensor are too expensive to be practically employed. Thus, estimation methods for vehicle body slip angle have been extensively studied during the last few decades, and most of them are based on bicycle model [2-3]; however, such model suffers from model uncertainty problems because it includes uncertain parameters, for example, tire cornering stiffness. Other kinds of prevailing sensors such as camera, although demonstrated high accuracy on position estimation for lane keeping [4], are seldom utilized for EV motion control due to their low throughput characteristic and the image processing often takes time. On the other hand, in contradiction to vehicle bicycle model, visual model is purely based on simple geometry which contains much fewer uncertainties and the estimation results can hence be more robust against bicycle model uncertainty. In addition, vision system can provide redundant information in addition to other onboard sensors; fault detection and fault tolerance control can then be applied.

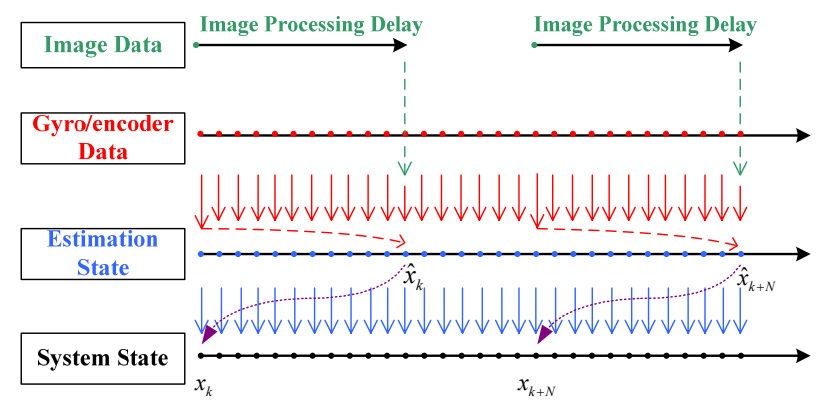

Fig. 1. System time sequence diagram.

As stated above, normal cameras are not fast enough for electric vehicle motion estimation and control. If the updating rate can be boosted, information from camera can be adopted for electric vehicle motion control that needs fast sensor feedback. In addition, the performances of lane keeping and collision avoidance systems can be enhanced for traditional vehicles [4-5].

In this research, traditional bicycle model is augmented with a visual model, and the new system has four states with three of them available from sensor/image processing program. Bicycle model uses signal from gyro sensor, steering angle and wheel speed encoders; visual model uses the information calculated from the onboard vision system. The sampling sequence diagram is shown in Fig. 1. Obviously, data from visual system and from gyro/encoders cannot be fused directly because of sampling time mismatch. Two solutions can be employed, namely, increase the overall sampling period to fit the longest one, and use multi-rate estimation method. These two methods will be further illustrated in the following sections.

\section{COMBINED VehicLE/Vision SySTEM Modeling AND EXPERIMENT SETUP}

\section{A. Bicycle Model}

Vehicle dynamics is highly non-linear, and it is impossible to describe vehicle motion precisely. Although there are complex models with high accuracy, from the perspective of implementation, simple linear model is preferable. Bicycle model with two degree of freedom, also known as single track model, is widely used in vehicle state estimation and motion control systems. This model is illustrated in Fig. 2, and the governing state space equation is given as (1). Detailed derivations and explanations can be found in [2-3]. 


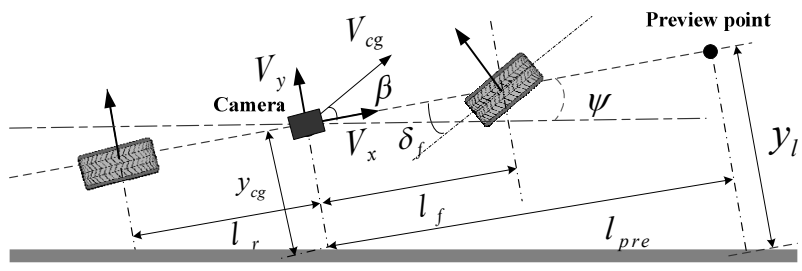

Fig. 2. Bicycle model and vision model.

$$
\begin{aligned}
& \dot{x}=A \cdot x+B \cdot u+w \\
& y=C \cdot x+v
\end{aligned}
$$

where

$$
\begin{aligned}
& x=\left[\begin{array}{ll}
\beta & \gamma
\end{array}\right]^{T}, \quad u=\delta_{f}, \quad y=\gamma, \\
& A=\left[\begin{array}{cc}
-\frac{c_{f}+c_{r}}{m V_{x}} & -1-\frac{c_{f} l_{f}-c_{r} l_{r}}{m V_{x}{ }^{2}} \\
-\frac{c_{f} l_{f}-c_{r} l_{r}}{I} & -\frac{c_{f} l_{f}^{2}-c_{r} l_{r}^{2}}{I V_{x}}
\end{array}\right], \\
& B=\left[\begin{array}{ll}
\frac{c_{f}}{m V_{x}} & \frac{c_{f} l_{f}}{I}
\end{array}\right]^{T}, \quad C=\left[\begin{array}{ll}
0 & 1
\end{array}\right] .
\end{aligned}
$$

\section{B. Vision System Model}

While bicycle model is independent of road reference, vision model contains geometric information by considering vehicle and lane/road together. Vision model is also shown in Fig. 2, and the gray borders are lane makers. $l_{\text {pre }}$ is a fixed preview distance need to be calibrated beforehand. In this model, it is assumed that the vehicle is travelling along a straight road with clear lane markers; the camera is equipped at the CoG (center of gravity) of the vehicle and can detect lane boundaries in real time. The lane function can be obtained in the coordinate with camera/vehicle as origin, and then, lateral distance offset $y_{l}$ at the preview point, as well as the heading angle $\psi$ can be calculated. $y_{c g}$ is the lateral offset at vehicle $\mathrm{CoG}$.

To derive vision model, $\psi$ and body slip angle $\beta$ are assumed to be small. Referring to Fig. 2, $y_{l}$ can be approximately expressed as (2);

$$
y_{l}=y_{c g}+l_{p r e} \cdot \psi
$$

The derivative of $y_{c g}$, i.e., the lateral speed at $\mathrm{CoG}$ is given by (3):

$$
\begin{aligned}
\dot{y}_{c g} & =V_{c g} \cdot \sin (\beta+\psi) \\
& =V_{x} \cdot(\beta+\psi)
\end{aligned}
$$

Equation (4) can be obtained by taking the derivative of (2) and substituting (3) into it:

$$
\dot{y}_{l}=V_{x} \cdot \beta+l_{p r e} \cdot \gamma+V_{x} \cdot \psi
$$

From (4), it can be known that, the derivative of offset at the preview point, i.e., lateral speed at that point, comprises three parts: the lateral speed of $\mathrm{CoG}$, the components of yaw rate, and the component of longitudinal speed (resulted from vehicle heading angle). Heading angle $\psi$ can be simply modeled as integration of yaw rate as (5).

$$
\dot{\psi}=\gamma
$$

It should be noticed that, although curved road is not considered here, models can still be generated in the same manner by taking curvature into account, i.e., this research can be expanded to all the roads with lane.

\section{Combined Model}

The combined model can also be given in continuous state space form as (1) with description in (6); the first two states are from bicycle model and the latter two are from the visual model. Clearly, the vision model is much simpler than the bicycle one. In the combined model, available system outputs are yaw rate, vehicle heading angle and lateral offset with reference to lane at the preview point. It should be pointed out that the first two states and the second two are updated at different sampling rate.

$$
\begin{gathered}
x=\left[\begin{array}{cccc}
\beta & \gamma & \psi & y_{l}
\end{array}\right]^{T}, \quad u=\delta_{f}, \quad y=\left[\begin{array}{ccc}
\gamma & \psi & y_{l}
\end{array}\right]^{T}, \\
A=\left[\begin{array}{cccc}
-\frac{c_{f}+c_{r}}{m V_{x}} & -1-\frac{c_{f} l_{f}-c_{r} l_{r}}{m V_{x}{ }^{2}} & 0 & 0 \\
-\frac{c_{f} l_{f}-c_{r} l_{r}}{I} & -\frac{c_{f} l_{f}^{2}-c_{r} l_{r}^{2}}{I V_{x}} & 0 & 0 \\
0 & 1 & 0 & 0 \\
V_{x} & l_{p r e} & V_{x} & 0
\end{array}\right], \\
B=\left[\begin{array}{cccc}
\frac{c_{f}}{m V_{x}} \frac{c_{f} l_{f}}{I} & 0 & 0
\end{array}\right]^{T}, \quad C=\left[\begin{array}{llll}
0 & 1 & 0 & 0 \\
0 & 0 & 1 & 0 \\
0 & 0 & 0 & 1
\end{array}\right] .
\end{gathered}
$$

\section{Experiment Setups}

The experiment vehicle used in this research is an inwheel-motor EV as in Fig. 3 (a). The prototype is COMS produced by Toyota Auto Body, and it was reengineered by our lab for capacitor research and motion control [11].

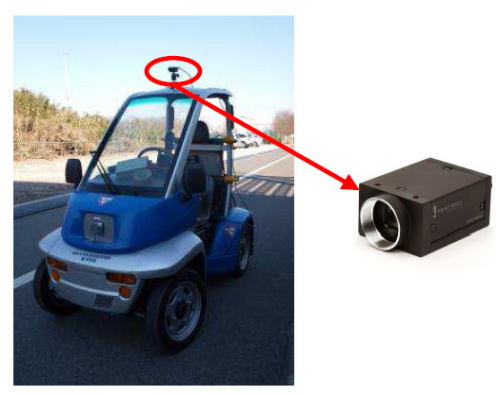

(a) Experimental in-wheel-motor EV. 


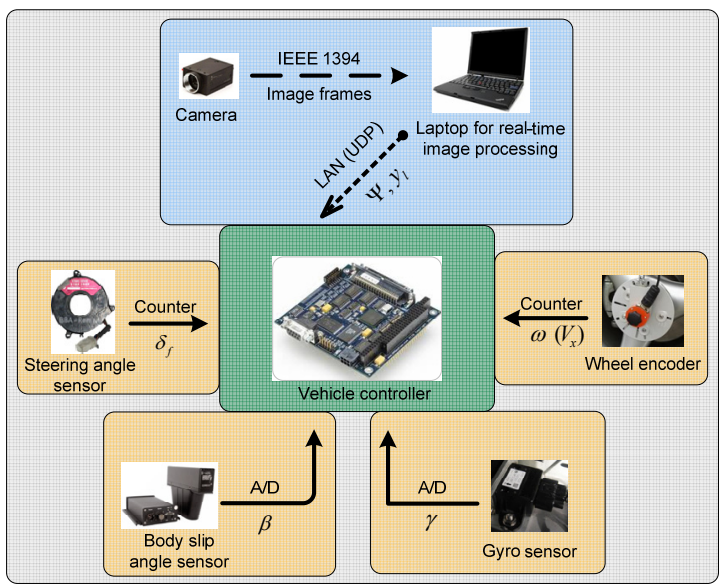

(b) Sensor configuration of COMS.

Fig. 3. Experimental vehicle and sensor configurations.

Fig. 3 (b) briefly demonstrates the sensor configurations of this vehicle. The vehicle controller is a PC104 embedded computer with ART-Linux system, and the program is configured to run at the speed of one millisecond per cycle. In addition to the central computer, four $\mathrm{A} / \mathrm{D}$ converters and two counter boards are equipped for sensor signal reading. Gyroscope is installed in the vehicle CoG, and provides yaw rate signal to the A/D board; steering angle and wheel speed encoders send data to the counter board. Besides, to verify estimation results, a non-contact optical sensor S-400 made by Corrsys-Datron is also equipped for body slip angle acquisition. Vision system includes a camera and an image processing laptop; the camera is Grasshopper produced by Point Gray, and it is installed on the top of the vehicle with a tilting angle of 8 degree and a preview distance of $5.135 \mathrm{~m}$. The frame rate of the camera is set to $30 \mathrm{fps}$.

Images captured by the camera are then grasped by a CARDBUS frame grabber and processed by a laptop with image processing algorithms in Linux environment. The image processing time varies from $8 \mathrm{~ms}$ to $25 \mathrm{~ms}$ which depends on the CPU load and the incoming images; for later data processing benefits, a delay function is implemented to make this time constant $(30 \mathrm{~ms})$. The final outputs from vision system are the estimated $\psi$ and $y_{l}$, and they are sent to vehicle controller via LAN cable using UDP protocol (the time for data transmission is neglected).

\section{REAL-TIME IMAGE PROCESSING FOR LANE DETECTION AND LOCATION}

Body slip angle estimation needs to fuse vision information with other sensor signals in real-time, thus, on-line image processing is indispensable. In this research, image processing is implemented in $\mathrm{C}++$ with OpenCV library and the library released by Point Grey Research. OpenCV is Open Source Computer Vision for short and it is a free library initially developed by Intel which can facilitate vision related development [6]. Image processing in this research generally consists of two parts: coordinate mapping and mapped images

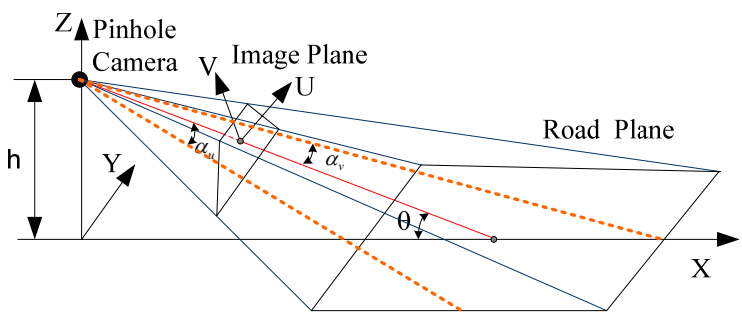

Fig. 4. Geometric relationship between camera and road.

processing. Coordinate mapping removes the perspective effect geometrically; image processing extracts lane makers from road, and gives the lane equation with reference to the vehicle coordinate.

\section{A. Mapping from Image to Real World}

Camera maps the 3-D world onto its 2-D image view; however, this kind of mapping makes it obscure to know the exact distance information between two pixels in the image plane. Since common road can be simplified as plane, such kind of perspective effect can be removed based on geometry relationships.

Fig. 4 shows the geometric relationships among pinhole camera model, image plane and road plane. In Fig. $4, h$ is height of camera, $f$ is focal length, $\theta$ is camera tilting angle, $\alpha_{v}$ and $\alpha_{u}$ are the angle of view in vertical and horizontal axis of image planes respectively. To find the mapping matrix from image coordinate to road coordinate, one typical method is to calibrate the camera model which is composed of both intrinsic and extrinsic parameters [10]. While this method needs complex calibration, another more straightforward method can get road coordinates from image pixel positions by deriving functions based on geometric relationships [12, 15]. For better demonstration, Fig. 4 is reconstructed as Fig. 5 viewing from $U$ axis and $V$ axis of image plane.
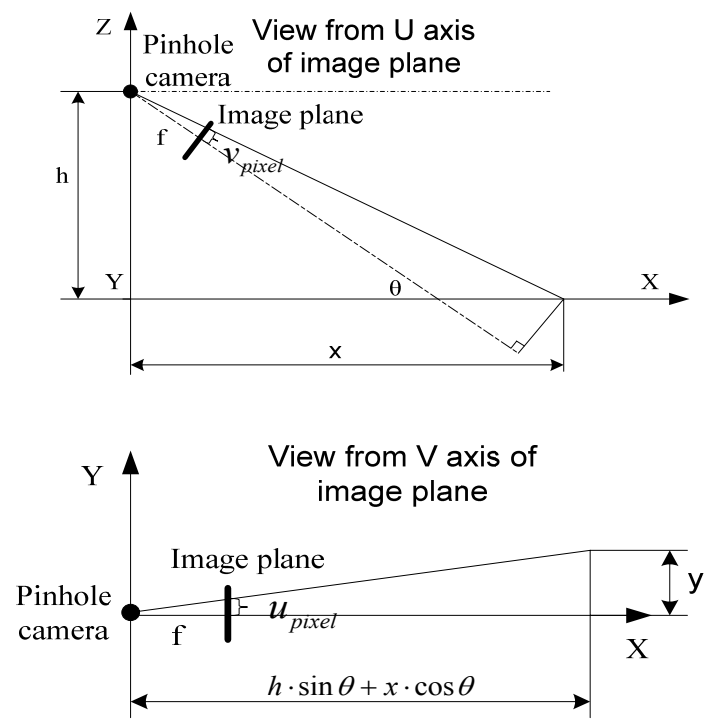

Fig. 5. View from different image plane axis. 

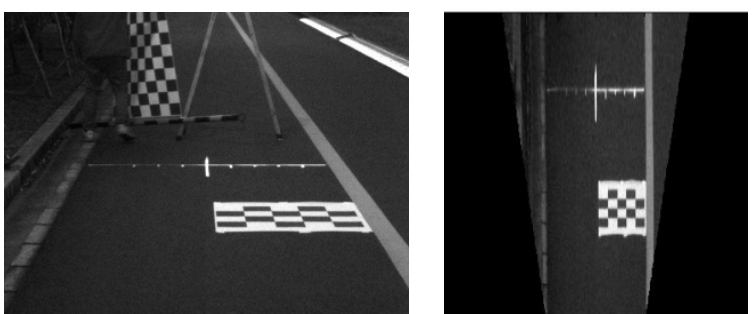

Fig. 6. Original and mapped view of the road.

Let's analyze one arbitrary pixel $\left(u_{\text {pixel }}, v_{\text {pixel }}\right)$ in the image plane and its projection $(x, y)$ on road plane. Assume the resolution of image is $m$ by $n$, equation (7) that mapping road coordinate to pixel coordinate can be derived.

$$
\begin{aligned}
& u_{\text {pixel }}=\frac{m-1}{2} \cdot\left(1+\frac{h-x \cdot \tan \theta}{h \cdot \tan \theta+x} \cdot \cot \alpha_{v}\right)+1 \\
& v_{\text {pixel }}=\frac{n-1}{2} \cdot\left(1-\frac{y}{h \cdot \sin \theta+x \cdot \cos \theta} \cdot \cot \alpha_{u}\right)+1
\end{aligned}
$$

From the above two equations, for any pixel in the image plane, the corresponding road coordinate can be calculated, and vice versa.

\section{B. Lane Extraction and Detection}

Thanks to the strong brightness contrast between road and lane, they can be distinguished with suitable algorithm. Here, LoG (Laplacian of Gaussian) method is implemented for lane center extraction, and it is an algorithm that performs Laplacian and Gaussian in a one step manner. Fig. 7 (b) illustrates the LoG results. RANSAC is employed among the candidate white points in Fig. 7 (b). This algorithm randomly selects smallest data set and finds how many data in total fit the model with the calculated parameters within a given tolerance; if the fitting ratio is large enough, it ends with success, otherwise proceeds till a specified number of trials. The red line in Fig. 7 (c) shows lane finding result. Detailed description can be found in [5].

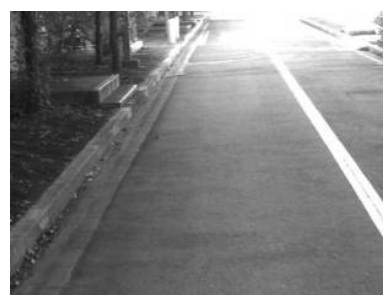

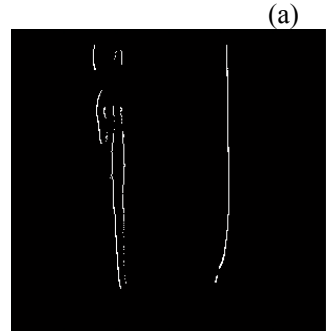

(b) Binarized image

Fig. 7. Image processing results.

\section{IV.}

\section{KALMAN Filter DESIGN FOR VEHICLE BODY SLIP} ANGLE ESTIMATION

Typical continuous state space equation has the form of (1); in this application, vehicle speed is not constant, and hence the discretized system matrix are designed to be time varying as in (8); $T_{s}$ is the sampling time. Fig. 8 describes the general structure of Kalman filter.

$$
\begin{aligned}
& x(k+1)=A_{d}(k) \cdot x(k)+B_{d}(k) \cdot u(k)+w(k) \\
& y(k)=C_{d}(k) \cdot x(k)+v(k)
\end{aligned}
$$

where

$$
\begin{aligned}
& A_{d}(k)=e^{A \cdot T s}, \quad B_{d}(k)=\int_{0}^{T s} e^{A \cdot \tau} \cdot B d \tau, \\
& C_{d}(k)=C .
\end{aligned}
$$

Discrete Kalman filter based on combined model (6) can be constructed in the form of (8). However, in case of measurement delay, the system output equation becomes (9).

$$
y(k)=C_{d}\left(k-n_{d}\right) \cdot x\left(k-n_{d}\right)+v\left(k-n_{d}\right)
$$

where $T_{d}$ is the time delay, and $n_{d}=T_{d} / T_{s} ; T_{d}$ is $33 \mathrm{~ms}$, and $T_{s}$ is $1 \mathrm{~ms}$ in this research.

From (9), it is known that, unlike normal system, the measurements are updated at time $k-n_{d}$, but are not available until time $k$. To see the effect of delay on estimation results, a simple simulation is conducted with the model in (6) using Kalman filter: with the same Kalman gain, the RMSE of body slip angle estimation is 0.0058 rad without delay, and goes up to $0.01176 \mathrm{rad}$ in the case of visual information delay. Therefore, estimation accuracy will be deteriorated without considering time lag.

Augmented-state method is employed to deal with delay issue [14]. The basic idea of this method is to include delayed states into the state space equation. First, considering information from camera has a one step delay, (10) can be defined:

$$
\bar{\psi}(k+1)=\psi(k), \quad \bar{y}_{l}(k+1)=y_{l}(k)
$$

Then, the combined system model in (6) can be discretized to (8) with (10) as additional states, and (11) gives description for the new system.

With the above transformation, delayed vision information is augmented to the original system. As this system is observable, current vision information can be estimated with Kalman filter based on previous ones.

Another problem is, as mentioned before, sampling periods of the system are not consistent; in (11), the first two states and the following four states have different sampling frequencies. Therefore, two solutions are adopted in this section, namely, single-rate and multi-rate Kalman filter. 


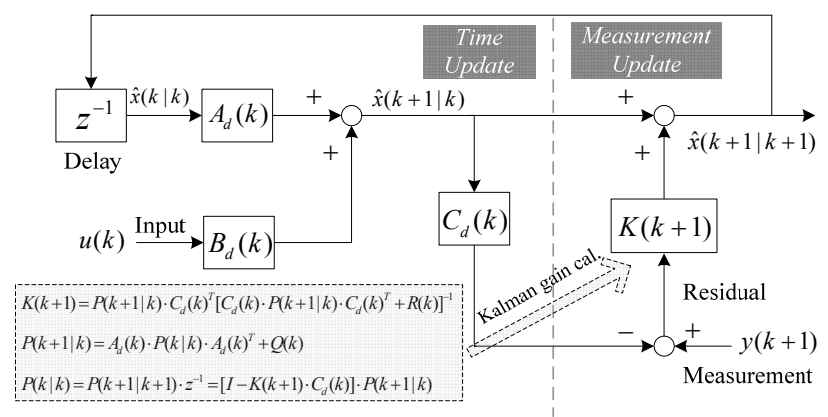

Fig. 8. General Kalman filter diagram.

$$
\begin{aligned}
& x^{a}(k)=\left[\beta(k), \gamma(k), \psi(k), y_{l}(k), \bar{\psi}(k), \bar{y}_{l}(k)\right]^{T}, \\
& u(k)=\delta_{f}(k), \quad y^{a}(k)=\left[\gamma(k), \bar{\psi}(k), \bar{y}_{l}(k)\right]^{T},
\end{aligned}
$$

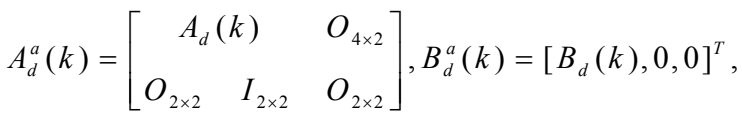

$$
\begin{aligned}
& C_{d}^{a}(k)=C_{d}^{a}=\left[\begin{array}{cccccc}
0 & 1 & 0 & 0 & 0 & 0 \\
0 & 0 & 0 & 0 & 1 & 0 \\
0 & 0 & 0 & 0 & 0 & 1
\end{array}\right] .
\end{aligned}
$$

\section{A. Single-rate KF based on delay-augmented system}

Single-rate Kalman filter simply adapt the sampling rate of high speed sensor to the low speed one. In this study, to unify the sampling rate of gyroscope, encoders and camera, system sampling rate is set to adapt camera. The following work is the same with traditional Kalman filter design which can be found in many literatures [7, 9].

Although the traditional Kalman filter can be employed by making the sampling rate adjustment, other problems are arisen, for example, measurements from high sampling rate sensors cannot be fully utilized and hence deteriorate the accuracy of sensor feedback (this can be observed from the experimental results in section V); the estimated body slip angle is updated every $33 \mathrm{~ms}$ which is not fast enough for general motion control applications [14].

\section{B. Multi-rate KF based on delay-augmented system}

Compared with single-rate KF, the prediction part of Multirate $\mathrm{KF}$ is the same, and the main differences between the two methods are: 1) the continuous state space equations are first discretized using the fast sampling time, i.e., $T s$ is set to $1 \mathrm{~ms}$; 2) the correction part of Multi-rate KF needs to be modified during inter-samplings. Assume the camera measurement sampling period is $T_{c}$, and during the time intervals of $n \cdot T_{c}$ ( $n$ is an integer), there is no sensor updates from vision system, so the correction of Kalman filter is only based on yaw rate from gyro sensor; another interpretation can be: pseudocorrection is implemented using estimated visual information (equal to prediction) while real-correction is done with yaw rate feedback. The state estimation equation for multi-rate Kalman filter is shown in (12).

$$
\begin{aligned}
& \hat{x}^{a}(k+1 \mid k+1)= \\
& \hat{x}^{a}(k+1 \mid k)+\tilde{K}(k+1) \cdot\left[\tilde{y}^{a}(k+1)-C_{d}^{a} \cdot \hat{x}^{a}(k+1 \mid k)\right]
\end{aligned}
$$

where

$$
\tilde{y}^{a}(k+1)=\left\{\begin{array}{l}
[\gamma(k+1), 0,0)]^{T}, \text { if } k+1 \neq n \cdot\left(T_{C} / T_{S}\right), \\
\left.\left[\gamma(k+1), \bar{\psi}(k+1), \bar{y}_{l}(k+1)\right)\right]^{T}, \text { if } \quad k+1=n \cdot\left(T_{C} / T_{S}\right) .
\end{array}\right.
$$

Kalman gain $\tilde{K}(k+1)$ in (12) changes following $\tilde{P}(k+1 \mid k)$, which is given by the below equation:

$$
\begin{aligned}
& \tilde{P}(k+1 \mid k)=A_{d}^{a}(k) \cdot \tilde{P}(k \mid k) \cdot A_{d}^{a T}(k)+Q(k) \\
& =A_{d}^{a}(k) \cdot\left(\left[I-\tilde{K}(k) \cdot \tilde{C}_{d}^{a}(k)\right] \cdot \tilde{P}(k \mid k-1)\right) \cdot A_{d}^{a T}(k)+Q(k)
\end{aligned}
$$

where

$$
\tilde{C}_{d}^{a}(k)=\left\{\begin{array}{l}
{\left[\begin{array}{llllll}
0 & 1 & 0 & 0 & 0 & 0 \\
0 & 0 & 0 & 0 & 0 & 0 \\
0 & 0 & 0 & 0 & 0 & 0
\end{array}\right], \text { if } k \neq n \cdot\left(T_{c} / T_{S}\right),} \\
{\left[\begin{array}{llllll}
0 & 1 & 0 & 0 & 0 & 0 \\
0 & 0 & 0 & 0 & 1 & 0 \\
0 & 0 & 0 & 0 & 0 & 1
\end{array}\right], \text { if } k=n \cdot\left(T_{c} / T_{S}\right) .}
\end{array}\right.
$$

The above equation illustrate that the Kalman gain is switching between two situations, namely, with and without visual updates. In other words, full measurement update is done when there are visual feedbacks, and partial measurement update is done during vision inter-samplings.

\section{SimUlations AND EXPERIMENT VERIFICATIONS}

\section{A. Simulations}

Simulations are conducted to verify the proposed multi-rate Kalman filter. The vehicle is assumed to run at the speed of $30 \mathrm{~km} / \mathrm{h}$, and to clearly demonstrate the effectiveness of the proposed method, vehicle model and Kalman filter model are made different from each other: the cornering stiffness and the mass of the vehicle plant are set 5 times and 2 times bigger than the estimated ones respectively. For comparison, bicycle model based two states Kalman filter and combined four states Kalman filter with $33 \mathrm{~ms}$ sampling intervals (single-rate KF) are also included.

From Fig. 9, it can be observed that the estimation result of the two states bicycle model (light blue line) cannot track true value (green line) well due to the model discrepancy; the four states Kalman filter (blue line) can improve estimation accuracy by augmenting bicycle model with the vision model, however, body slip angle is only updated every $33 \mathrm{~ms}$; the four state multi-rate Kalman filter (red line), on the other hand, has yaw rate measurement update during the camera sampling intervals, and hence can be more accurate than the other two methods; in addition, it can be applied to motion control applications which need fast signal feedback. In general, multi-rate KF is more robust against model error. 


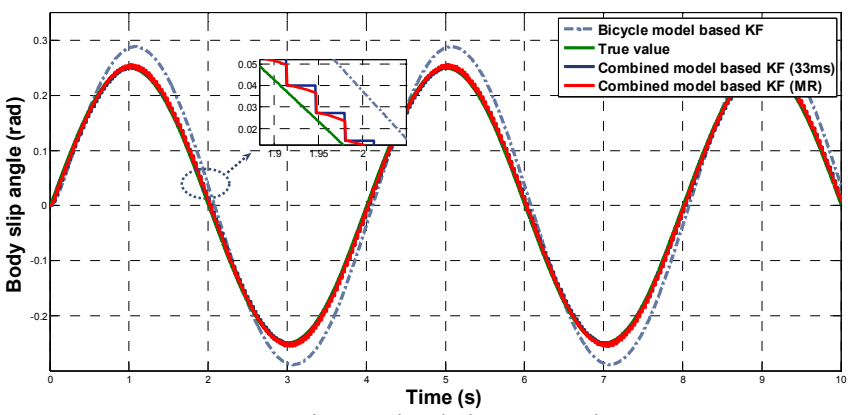

Fig. 9. Simulation comparison.

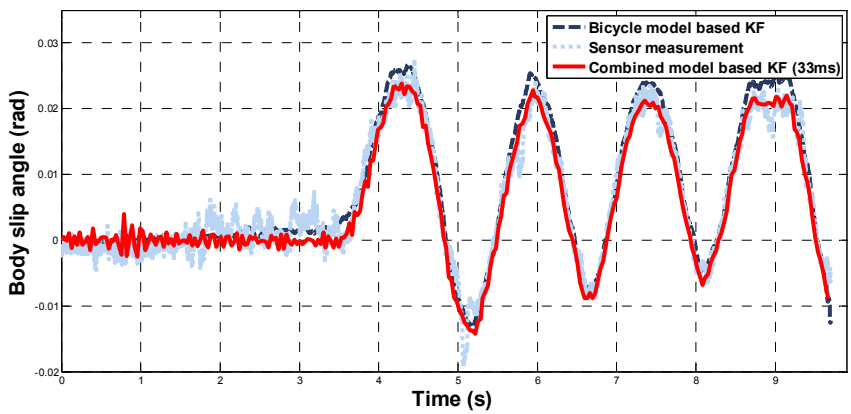

(a) Single-rate combined model vs. bicycle model.

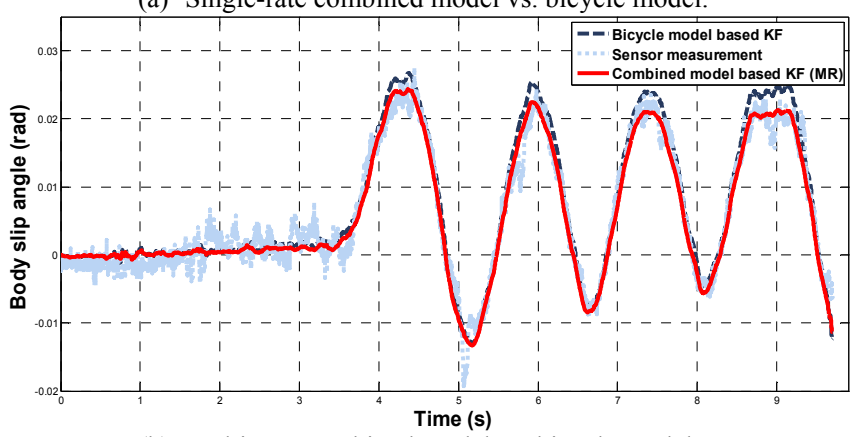

(b) Multi-rate combined model vs. bicycle model.

Fig. 10. Estimation comparison based on experiment data.

\section{B. Experiments}

Experiments are conducted with the experimental electric vehicle COMS introduced in section II. The vehicle was given a sine steering input on a road with lane markers, and the vehicle speed is varying from $10 \mathrm{~km} / \mathrm{h}$ to $20 \mathrm{~km} / \mathrm{h}$.

Similar to the simulation setting above, model mismatch is also applied to the Kalman filter model. Fig. 10 (a) gives comparison results of bicycle model-based Kalman filter and combined four states-based single-rate Kalman filter. Due to the model discrepancy, bicycle model-based result (blue line) cannot track the true value (dotted light blue line) in some situations; in contrary, the augmented single-rate estimator (red line) performs much better. However, this single-rate estimator gets sensor feedback every $33 \mathrm{~ms}$, and hence cannot fully utilize sensor information; as a result, vibration occur in some parts of the estimation result. In Fig. 10 (b), multi-rate Kalman filter result (red line) that can be updated every $1 \mathrm{~ms}$ is demonstrated; as can be observed, it is much more accurate compared with bicycle model-based Kalman filter estimation result (blue line), and is smoother than the result of singlerate Kalman filter. Compared to bicycle model-based Kalman filter, RMSE of the single-rate four state Kalman filter decreases $16 \%$, and RMSE of the multi-rate four state Kalman filter decreases $28 \%$.

\section{CONCLUSION AND FUTURE WORKS}

In this paper, a combined vehicle and visual model is explained; real-time image processing techniques for lane detection and location are introduced; single-rate Kalman filter which has a low updating rate is first developed, and multi-rate Kalman filters that can be updated every $1 \mathrm{~ms}$ are designed to estimate vehicle body slip angle for EV motion control. The further works of this research are: state estimation for varying sampling system (image processing time is not constant); multi-rate control using low frequency estimation result for EV control. In addition, in case of visual system malfunction, fault detection and fault tolerance control methods should be investigated.

\section{REFERENCES}

[1] Yoichi Hori, "Future Vehicle driven by Electricity and Control Research on 4 Wheel Motored 'UOT March II'", IEEE Transactions on Industrial Electronics, Vol.51, No.5, pp.954-962, 2004.10.

[2] C. Geng, L. Mostefai, M. Denai and Y. Hori, "Direct Yaw Moment Control of an In-Wheel-Motored Electric Vehicle Based on Body Slip Angle Fuzzy Observer", IEEE Transactions on Industrial Electronics, Vol.56, No.5, MAY2009.

[3] T. Hiraoka, H. Kumamoto, O. Nishihara, "Sideslip angle estimation and active front steering system based on lateral acceleration data at centers of percussion with respect to front/rear wheels", JSAE Review, Vol.25, No.1, pp.37-42,2004

[4] Jin-Chuan Hsu, M. Tomizuka, "Analyses of vision-based lateral control for automated highway system", Vehicle system dynamics, 1998.

[5] Joel C. McCall and Mohan M. Trivedi, "Video Based Lane Estimation and Tracking for Driver Assistance: Survey, System, and Evaluation", IEEE Tran. on ITS, Vol.7, pp.20-37, 2006.

[6] G. Bradski, A. Kaehler, "Learning OpenCV: Computer Vision with the OpenCV Library", O'Reilly,Cambridge, MA, 2008.

[7] Greg Welch, Gary Bishop, "An Introduction to the Kalman Filter".

[8] S. On, and Y. Hori, "Development of a Novel Instantaneous Speed Observer and its Application to the Power-Assisted Wheelchair Control", Proc. 4th International Conference on Power Electronics and Motion Control, 2004.8.14-16, Xi'an, China, 2004.

[9] L. Chu, Y. Zhang, M. Xu, Y. Shi, and O. Yang, "Design of a robust side slip angle observer using adaptive Kalman filter", Computer Design and Applications (ICCDA), 2010 International Conf.on, 2010.

[10] M. Chen, T. Jochem, D. Pomerleau, "AURORA: A Vision-Based Roadway Departure Warning system", Proceedings of IEEE International Conf. on Intelligent Robots and Systems, Pittsburgh, PA, August, 1995, pp. 243-248, 1995.

[11] K. Kawashima, T. Uchida, and Y. Hori, "Manufacturing of Small Electric Vehicle driven only by Electric Double Layer Capacitors for Easy Experiment of Vehicle Motion Control", Electric Vehicle Symposium 21, 2005.4.

[12] M. Bertozzi and A. Broggi, "GOLD: a Parallel Real-Time Stereo Vision System for Generic Obstacle and Lane Detection", IEEE Transactions on Image Processing 7, pp. 62-81, January 1998.

[13] Dejun Yin, Yoichi Hori, "A Novel Traction Control for EV Based on Maximum Transmissible Torque Estimation", IEEE Transactions on Industrial Electronics, Vol. 56, No.6, 2009.

[14] S. Challa, R. Evans and X. Wang, "A Bayesian solution and its approximations to out-of-sequence measurement problems", Journal of Information Fusion, Vol. 4, Issue 3, pp. 185-199, September 2003.

[15] Muad, A.M. Hussain, A. Samad, S.A. Mustaffa, M.M. Majlis, B.Y., "Implementation of inverse perspective mapping algorithm for the development of an automatic lane tracking system", TENCON 2004, Vol. 1, pp. 207-210, 2004. 